\title{
Immunohistochemical detection of GluA1 subunit of AMPA receptor in the rat nucleus accumbens following cocaine exposure
}

\author{
Wen Ting Cai, Joonyeup Han, Wha Young Kim*, and Jeong-Hoon Kim* \\ Department of Physiology, Graduate School of Medical Sciences, Yonsei University College of Medicine, Seoul 03722, Korea
}

\section{ARTICLE INFO}

Received November 6, 2020

Revised November 20, 2020

Accepted November 23, 2020

*Correspondence

Wha Young Kim

E-mail:maru0222@yuhs.ac

Jeong-Hoon Kim

E-mail: jkim1@yuhs.ac

Key Words

Cocaine

Glutamate receptor

Nucleus accumbens

Tissue fixation

\begin{abstract}
AMPA) receptors are differentially regulated in the nucleus accumbens (NAcC) of the brain after cocaine exposure. However, these results are supported only by biochemical and electrophysiological methods, but have not been validated with immunohistochemistry. To overcome the restriction of antigen loss on the postsynaptic target molecules that occurs during perfusion-fixation, we adopted an immersion-fixation method that enabled us to immunohistochemically quantify the expression levels of the AMPA receptor GluA1 subunit in the NAcc. Interestingly, compared to saline exposure, cocaine significantly increased the immunofluorescence intensity of GluA1 in two sub-regions, the core and the shell, of the NAcc on withdrawal day 21 following cocaine exposure, which led to locomotor sensitization. Increases in GluA1 intensity were observed in both the extra-post synaptic density (PSD) and PSD areas in the two sub-regions of the NAcc. These results clearly indicate that AMPA receptor plasticity, as exemplified by GluA1, in the NAcc can be visually detected by immunohistochemistry and confocal imaging. These results expand our understanding of the molecular changes occurring in neuronal synapses by adding a new form of analysis to conventional biochemical and electrophysiological methods.
\end{abstract}

\section{INTRODUCTION}

It is well known that chronic exposure to psychomotor stimulants such as cocaine produces long-lasting neuronal plasticity in the brain reward circuit, including the nucleus accumbens (NAcc), which is a central neuronal substrate mediating the rewarding effects of drug abuse [1,2]. As the NAcc receives intense glutamatergic innervations from the cortical and limbic areas, including the prefrontal cortex, amygdala, and hippocampus [3], neuronal plasticity involving glutamate receptors is conspicuously evident at this site $[4,5]$. Among the diverse subtypes of glutamate receptors, ionotropic $\alpha$-amino-3-hydroxy-5-methyl-4-isoxazole propionic acid (AMPA) receptors are known to be critical for activity- dependent synaptic plasticity and they play an important role in cocaine-induced behavioral effects [6-8]. For example, it has been shown that behavioral sensitization to cocaine [9], and cueinduced cocaine seeking and its reinstatement $[6,10]$ require activation of intact AMPA receptors in the NAcc. Furthermore, it has also been shown that chronic exposure to cocaine regulates the potentiation levels of AMPA-mediated glutamatergic neurotransmission in the NAcc through up- and downregulation of AMPA receptor expression, depending on the period of withdrawal $[6,8,11]$.

Interestingly, the time-dependent plasticity of AMPA receptors in the NAcc has only been characterized by biochemical and electrophysiological measures $[8,12]$. The integration of im-

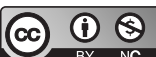

This is an Open Access article distributed under the terms of the Creative Commons Attribution Non-Commercial License, which permits unrestricted non-commercial use, distribution, and reproduction in any medium, provided the original work is properly cited. Copyright @ Korean J Physiol Pharmacol, pISSN 1226-4512, elSSN 2093-3827
Author contributions: W.T.C., W.Y.K., and J.-H.K. designed the experimental strategy and analyzed the data. W.T.C., J.H., and W.Y.K. performed all the experiments. W.T.C. and J.-H.K. wrote the manuscript. All authors commented on and approved the final manuscript. 
munohistochemistry and imaging techniques, such as confocal laser scanning microscopy, allows for sensitive visualization and quantification of protein expression in the region of interest [1315]. However, it has not yet been successfully applied to examine the differential expression of AMPA receptors in the NAcc. One of the difficulties of using immunohistochemistry to assess postsynaptic proteins such as AMPA receptors is that the antigenicity of these proteins tends to be reduced after tissue fixation. Conventional perfusion-fixation exposes tissues to an aldehyde that is usually sequestered in the cells, which causes changes in antigenic sites by cross-linking with proteins and results in a loss of antigenicity [15]. Therefore, this method has often yielded diffuse intracellular staining of the AMPA receptors and provides only a meager signal to distinguish the synaptic sites [16]. To address this issue, diverse methods have been attempted, such as microwave heating [17], pepsin pretreatment [18], and inhibition of proteolysis during fixation [14], and they each confer various advantages and disadvantages. Interestingly, a compromised weak immersion-fixation method has been recently introduced, which minimizes the exposure of tissues to aldehydes by altering the conventional paraformaldehyde (PFA)-based cardiac perfusion process. Using this technique, Notter et al. [15] successfully demonstrated the visualization of postsynaptic receptors as well as cytoplasmic cell markers, including molecules associated with presynaptic vesicles.

AMPA receptors comprise different combinations of four subunits (GluA1-4), that form a tetramer [19]. Among these, GluA1 is the most predominant subunit in neuronal synaptic and extrasynaptic AMPA receptors [20] and it plays a dominant role in activity-dependent recruitment of these receptors to synapses by controlling the levels of phosphorylation [19,21,22]. Furthermore, GluAl is known to play a particularly prominent role in cocaineseeking behavior [23] and is one of the major AMPA receptor subunits regulated by chronic cocaine exposure [8]. With these factors in mind, our aim in the present study was to localize and quantify post synaptic GluA1 in the NAcc, using a modified immersion-fixation immunohistochemical protocol and confocal imaging, to examine GluAl expression in this site after long-term withdrawal following chronic cocaine exposure.

\section{METHODS}

\section{Subjects and drugs}

Male Sprague-Dawley rats (6 weeks old) weighing 220-250 g on arrival were obtained from Orient Bio Inc. (Seongnam, Korea). They were housed three per cage in a 12-h light/dark cycle room (lights out at 8:00 PM), and all experiments were conducted during the day. Rats had access to water and food ad libitum at all times. All animal use procedures were conducted according to an approved Institutional Animal Care and Use Committee protocol (approval number: 2015-0271) of Yonsei University College of Medicine.

Cocaine hydrochloride (Belgopia, Louvain-La-Neuve, Belgium) was dissolved in sterile $0.9 \%$ saline to a final working concentration of $15 \mathrm{mg} / \mathrm{ml}$.

\section{Locomotor activity}

Locomotor activity was measured using a bank of 9 activity boxes $(35 \times 25 \times 40 \mathrm{~cm})$ (IWOO Scientific Co., Seoul, Korea) made of translucent Plexiglas. Each box was individually housed in a PVC plastic sound attenuating cubicle. The floor of each box consisted of 21 stainless steel rods ( $5 \mathrm{~mm}$ diameter), spaced $1.2 \mathrm{~cm}$ apart, center-to-center. Two infrared light photo beams (Med Associates, St. Albans, VT, USA) were positioned $4.5 \mathrm{~cm}$ above the floor and spaced evenly along the longitudinal axis of the box, to estimate horizontal locomotion. A single locomotor activity was only considered to be legitimate when a rat interrupted two beams consecutively, so as to avoid any possible confounding activities, such as grooming in a spot covering just a single beam.

\section{Immersion-fixation}

Rats were deeply anesthetized with intraperitoneal (IP) ketamine $(100 \mathrm{mg} / \mathrm{kg})$ and xylazine $(6 \mathrm{mg} / \mathrm{kg})$ prior to transcardial perfusion with ice-cold $10 \mathrm{mM}$ PBS (pH 7.4). The brain was quickly removed, and the tissues containing the NAcc area were immediately cut into blocks (with thickness of 9 to $10 \mathrm{~mm}$ ) on an ice-cold metal brain matrix. Then, the tissue blocks were immersed in ice-cold 4\% PFA solution in $10 \mathrm{mM}$ phosphatebuffered saline (PBS, pH 7.4) for $1 \mathrm{~h}$. After washing with $10 \mathrm{mM}$ PBS, the tissue blocks were transferred to $30 \%$ sucrose and stored at $4^{\circ} \mathrm{C}$ overnight. The next day, the tissue blocks were embedded and stored at $-80^{\circ} \mathrm{C}$.

\section{Immunohistochemistry}

Coronal brain sections $(40 \mu \mathrm{m})$ were processed from frozen brain tissue blocks on a cryostat, ranging from 0.7 to $2.2 \mathrm{~mm}$ from the bregma toward the anterior direction, which included the NAcc core and shell [24]. Then, free-floating sections were immersed for $1 \mathrm{~h}$ in $10 \mathrm{mM}$ PBS (pH 7.4) containing 5\% normal goat serum (Jackson ImmunoResearch Inc., West Grove, PA, USA) and $0.3 \%$ Triton X-100, followed by incubation overnight with anti-GluA1 antibody (rabbit, 1:1,000 [0.1 $\mu \mathrm{g} / \mathrm{ml}]$, Cat. No. Ab1504; Millipore, Burlington, MA, USA), diluted in 10 mM PBS containing $2 \%$ normal goat serum and $0.1 \%$ Triton $\mathrm{X}-100$ at $4^{\circ} \mathrm{C}$. Then, the sections were rinsed 3 times (10 min each) in $10 \mathrm{mM}$ PBS containing $0.1 \%$ Triton X-100 and incubated with the antirabbit IgG antibody coupled to Alexa Fluor 568 (goat, 1:1,000 [2 $\mu \mathrm{g} / \mathrm{ml}$, Cat. No. A11011; Invitrogen, Carlsbad, CA, USA) for 2 $\mathrm{h}$ at room temperature. The sections were then rinsed 3 times 
(10 min each) in $10 \mathrm{mM}$ PBS containing $0.1 \%$ Triton X-100 and mounted with Vectashield mounting medium (Vector Laboratories, Burlingame, CA, USA) under a coverslip. Double immunofluorescence staining was performed using a mixture of antiGluA1 (rabbit, 1:1,000 [0.1 $\mu \mathrm{g} / \mathrm{ml}]$, Cat. No. Ab1504; Millipore) with anti-post synaptic density (PSD) 95 (mouse, 1:1,000 [1 $\mu \mathrm{g} /$ ml], Cat. No. Ab2723; Abcam, Cambridge, UK) primary antibodies followed by a mixture of anti-rabbit IgG coupled to Alexa Fluor 568 (goat, 1:1,000 [2 $\mu \mathrm{g} / \mathrm{ml}$ ], Cat. No. A11011; Invitrogen) and anti-mouse IgG coupled to Alexa Fluor 488 (goat, 1:1,000 [2 $\mu \mathrm{g} / \mathrm{ml}]$, Cat. No. A11001; Invitrogen) secondary antibodies.

\section{Image analysis for quantification of GluA1 intensity}

All fluorescently labeled brain sections were examined under an LSM700 confocal laser scanning microscope (Carl Zeiss, Oberkochen, Germany) with diode lasers at 488 and $555 \mathrm{~nm}$. Images were obtained at a resolution of 1,024 × 1,024 pixels per $\mathrm{x}-\mathrm{y}$ plane, and stacks spaced by $0.5 \mu \mathrm{m}$ along the $z$-axis were acquired with 1 Airy unit of optical thickness using a 40x (or 63x) oil immersion objective (numerical aperture 1.4) depending on the purpose of the experiment. Scan averaging was set to 4 to improve the signal-to-noise ratio of each optical section. All images were acquired with identical settings for laser intensity, photomultiplier voltages, and scan speed.

On each section, one or two square regions from the core and shell, respectively, were randomly selected. The GluA1 intensity for a selected single square region $(101.52 \times 101.52 \mu \mathrm{m})$, which was obtained with raw image stacks, was quantified using ZEN image analysis software (Carl Zeiss). First, the brightest optical image stack along the $\mathrm{z}$-axis was designated as the reference image. Then, the analysis was performed with three consecutive images, including the reference itself and two adjacent images (above and below, spaced $0.5 \mu \mathrm{m}$ apart). The mean value of the GluA1 intensity readings obtained from these three images was used as a value representing the GluA1 intensity for the selected single region. This procedure normalizes the variability of GluA1 intensity due to different antibody penetration and light scattering effects along the depth of the tissue. In order to distinguish GluA1 expression in PSD from non-PSD areas, the intensity of co-localized PSD95 was also analyzed. Considering the spreading effect of fluorescence and the relatively small size of PSD (mean diameter of approximately $300-400 \mathrm{~nm}$ ) [25], we defined the PSD area as displaying a PSD95 intensity at least two times higher than the mean intensity of total PSD95, and the rest of the area (with a lower PSD95 intensity) as being extra-PSD.

The mean values of the GluA1 intensity from selected regions per slice were averaged for each experimental group and compared for PSD vs. extra-PSD areas, the core vs. shell of the NAcc, and saline vs. cocaine treatment groups, respectively.

\section{Design and procedures}

Upon arrival, all rats passed a week-long adaptation period to the new housing environment. They were divided into two groups and exposed daily to saline or cocaine $(15 \mathrm{mg} / \mathrm{kg}$, IP) for 7 consecutive days. Locomotor activity was measured on days 1 and 7 . The rats were first habituated to the activity boxes for $1 \mathrm{~h}$, and their locomotor activity was measured for $1 \mathrm{~h}$ immediately following saline or cocaine IP injections. To avoid any confounding effects of conditioning, the drugs were administered to the rats in different places (i.e., in the activity boxes for the first and the seventh injections and in their home cages for the other injections). The regimen of drug injection and dosages that we used here has previously been shown to produce locomotor sensitization $[26,27]$. Three weeks after the last injection (a 21-day period of withdrawal), all rats were perfused and their brains were removed for immersion-fixation. Brain sections obtained from both saline - and cocaine-exposed rats were stained simultaneously and mounted onto the same gelatin-coated slides. Using confocal imaging, the analyses of GluAl intensity in PSD and extra-PSD areas in the NAcc core and shell were conducted by a researcher who was blinded to the treatment group of the samples. A total of 8 rats were included for statistical analysis in this study, with no animals excluded.

\section{Statistical analyses}

Statistical analyses were performed using Sigma Plot version 12.0 (Systat Software, San Jose, CA, USA). The locomotor activity counts were analyzed with two-way repeated analysis of variance (ANOVA), while GluA1 intensities were analyzed with two-way ANOVA, followed by post-hoc Bonferroni comparisons. Differences between experimental conditions were considered statistically significant at $\mathrm{p}<0.05$.

\section{RESULTS}

\section{Differential GluA1 expression in the NAcc after cocaine exposure is detectable by immersion-fixation immunohistochemistry}

As it has been previously reported that repeated cocaine exposure induces AMPA receptor plasticity in the rat NAcc $[6,11,28]$, we used immunohistochemistry to examine and quantify GluA1 intensities at this site after a long-term withdrawal following chronic cocaine exposure. The experimental timeline depicting the cocaine injection scheme and the representative brain regions in which GluA1 intensity was measured are shown in Fig. 1.

Fig. 2 shows the locomotor activity of rats exposed to saline or cocaine, on days 1 and 7. The two-way repeated ANOVA analyses of these data revealed multiple effects of cocaine exposure $\left(\mathrm{F}_{1,6}\right.$ 


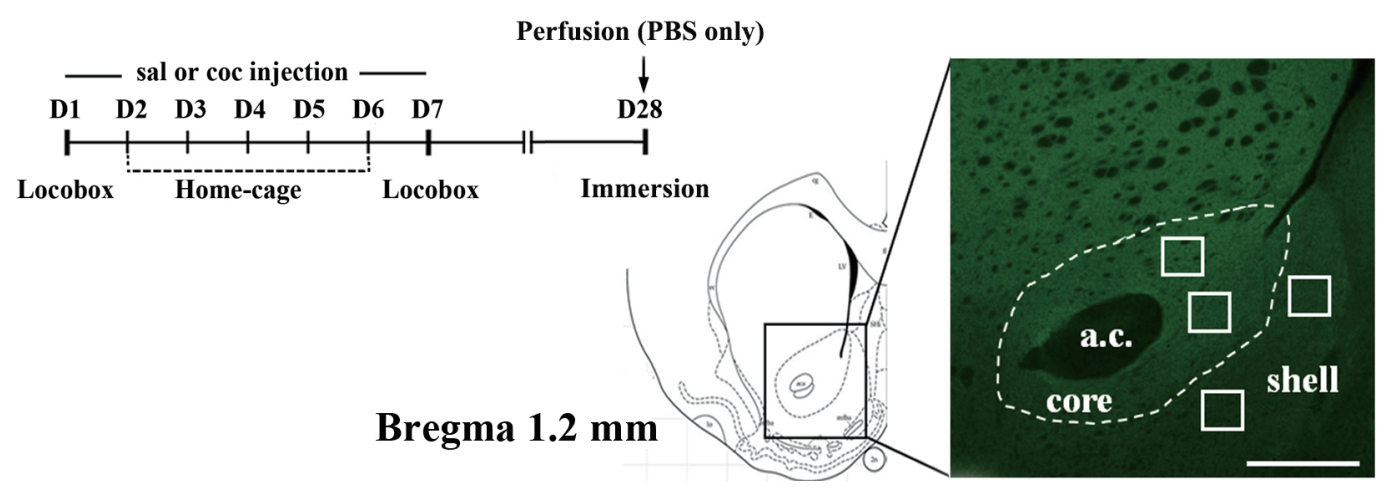

Fig. 1. Experiment outline. An experimental timeline indicating the cocaine sensitization scheme and the point at which immersion-fixation was performed. A schematic diagram of a matched coronal brain section (left) is adapted from the brain atlas of Paxinos and Watson [24], and a representative fluorescent image of the NAcc shows some of the sampled regions (white square boxes) selected for GluA1 analysis (right). Only one or two sample regions from both core and shell, respectively, were obtained from each slice of brain sections. The scale bar is $500 \mu \mathrm{m}$.

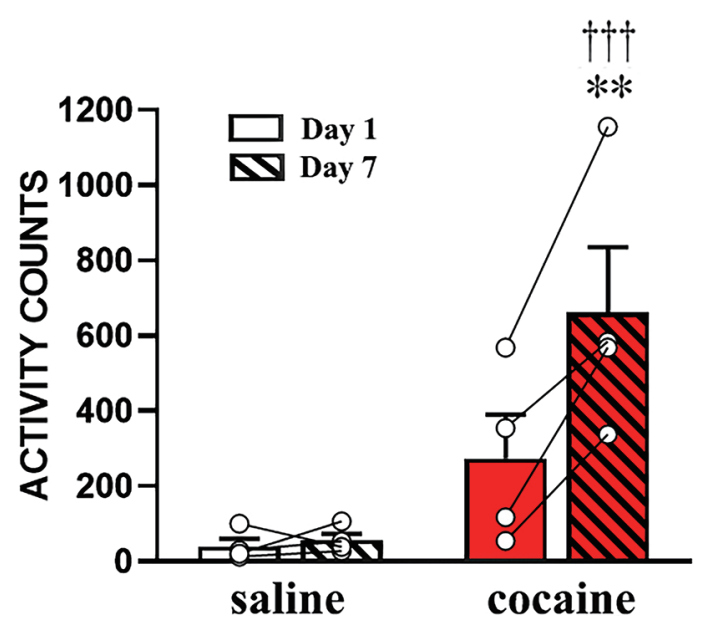

Fig. 2. Locomotor activity counts during exposure. All rats were habituated to the locomotor activity chamber for $1 \mathrm{~h}$ and their locomotor activity measured for an additional $1 \mathrm{~h}$ following their respective injections. Locomotor activity was measured only at day 1 and 7 as remaining injections were administered in the home cage (once daily, a total of 7 injections). Data are expressed as mean + standard error of the mean. ${ }^{* *} p<0.01$, significant differences compared to saline exposed animals. ${ }^{t+t} p<0.001$, significant difference at day 7 compared to day 1 in cocaine exposed group as revealed by post-hoc Bonferroni comparisons following two-way repeated ANOVA.

$=8.55, \mathrm{p}<0.05)$, duration $\left(\mathrm{F}_{1,6}=21.56, \mathrm{p}<0.01\right)$, and exposure $\times$ duration interactions $\left(\mathrm{F}_{1,6}=18.33, \mathrm{p}<0.01\right)$. As expected, rats with cocaine exposure showed higher locomotor responses than those exposed to saline, and they exhibited a significantly enhanced increase in locomotor responses on day 7 compared to day 1 ( $\mathrm{p}<$ 0.001 by post-hoc Bonferroni comparison), indicating that they developed locomotor sensitization to cocaine as a result of this drug treatment regimen.

On withdrawal day 21 after repeated exposure to saline or cocaine, the rats were perfused and the brain tissues processed for immunohistochemistry using the immersion-fixation method. Representative images, co-labeled with PSD95 and GluA1, of selected regions in the NAcc core and shell are shown in Fig. 3A. The relative values of GluA1 intensities between the PSD and extra-PSD areas in two different sub-regions of the NAcc are depicted in Fig. 3B. The two-way ANOVA analysis of these data revealed multiple effects due to cocaine exposure $\left(\mathrm{F}_{1,91}=9.22, \mathrm{p}=\right.$ 0.003 for the core; $\mathrm{F}_{1,91}=15.81, \mathrm{p}<0.001$ for the shell) and NAcc area $\left(\mathrm{F}_{1,91}=255.55, \mathrm{p}<0.001\right.$ for the core; $\mathrm{F}_{1,91}=204.91, \mathrm{p}<0.001$ for the shell). Post hoc Bonferroni comparisons of these data showed that GluAl intensity in both the core and the shell were significantly increased in cocaine- compared to saline-exposed rats, which were observed in both extra-PSD and PSD areas (Fig. $3 \mathrm{~B})$. It is interesting to note that GluAl intensities in the PSD areas were always higher than those in the extra-PSD areas, regardless of the sub-regions of the NAcc that were analyzed.

\section{DISCUSSION}

To our knowledge, the results of this study are the first to directly demonstrate that differential regulation of GluA1 expression in the NAcc can be visually detected and quantified using a combination of immunohistochemistry and confocal imaging techniques.

AMPA receptors are responsible for the majority of excitatory neurotransmissions in the brain and they contribute to changes in synaptic strength via receptor trafficking in and out of PSD areas $[22,29,30]$. It will therefore be very interesting to visually localize and quantify these receptors histologically. Although immunoelectron microscopy provides a more precise detection of the structure and chemical composition of PSD [31], it is not easily adopted in many laboratories due to certain challenges that are technically difficult compared to the relatively simple establishment of light microscopy-based techniques. As an alternative, confocal laser scanning microscopy can provide qualitative visualization of spatial segmentation to locate synaptic compartments in tissue sections [32]. However, as with other receptors residing 
A
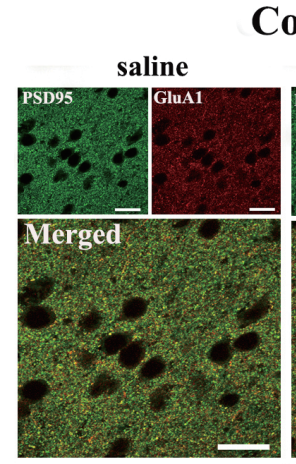

Core
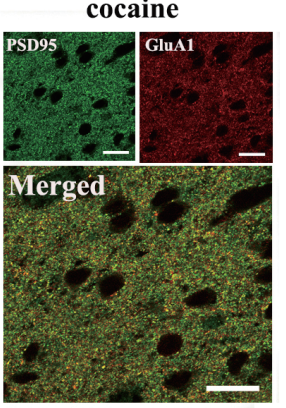

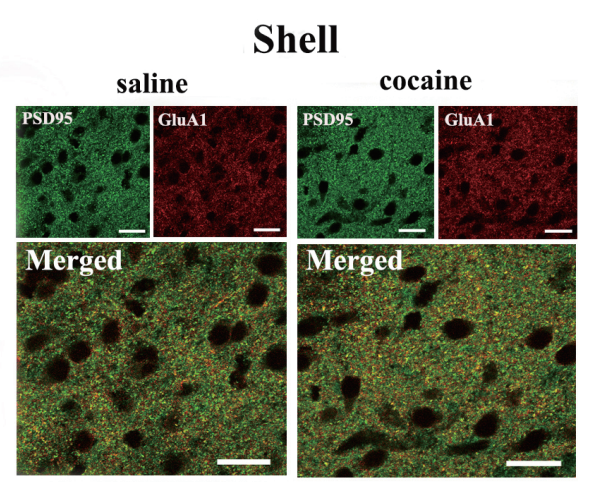

B

Core

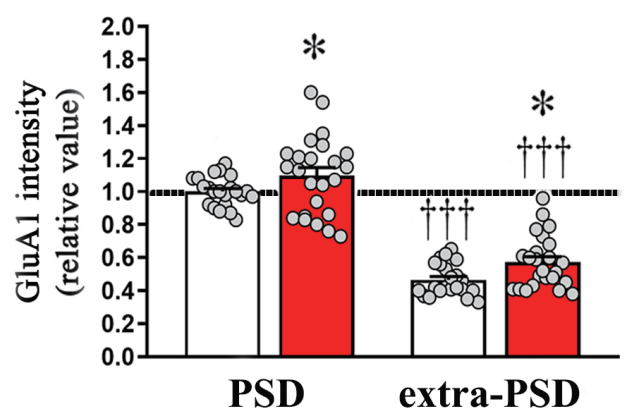

Shell

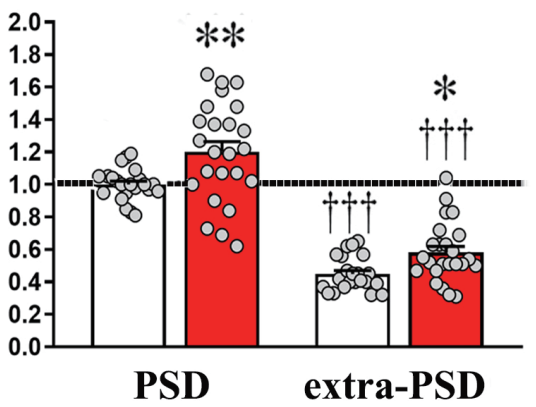

Fig. 3. Differential GluA1 expression in the NAcc after cocaine exposure is detectable using immersion-fixation immunohistochemistry. (A) Representative images showing co-localization of GluA1 and PSD95 in the NAcc core and shell, obtained with 1-h immersion-fixation on withdrawal day 21 after repeated exposure to saline or cocaine. Green and red indicates PSD95 and GluA1 expression, respectively, while yellow indicates colocalization of the two proteins. The scale bar is $20 \mu \mathrm{m}$. (B) Analysis of GluA1 intensity in the NAcc core and shell obtained with 1-h immersion-fixation on withdrawal day 21 after repeated exposure to saline or cocaine. Data are expressed as mean + standard error of the mean. relative to GluA1 intensity in the PSD area of the saline-exposed group. Symbols indicate significant differences as revealed by post-hoc Bonferroni comparisons following two-way ANOVA analysis. ${ }^{*} p<0.05,{ }^{* *} p<0.01$; significantly different compared to saline pre-exposed group within each area (PSD or extra-PSD). ${ }^{t+t} p$ $<0.001$; significantly different compared to PSD area. The total numbers of slices used in the analysis for the NAcc core and shell from 4 animals are 22 and 24 for saline- and cocaine-exposed rats, respectively. The sample numbers for PSD and extra-PSD are the same in all cases.

in the PSD area, AMPA receptors are difficult to visually localize and quantify, as it is hard to differentiate their specific loci in different sub-cellular areas. As mentioned earlier, this is mainly due to reduced antigenicity resulting from perfusion-fixation [16]. Although aldehydes typically cross-link proteins and thus block penetration of antibodies into the narrow synaptic cleft (as has been observed using conventional cardiac perfusion-fixation), others have previously shown that synaptic molecules can be successfully visualized with a protocol featuring a weak exposure to aldehyde, via immersion-fixation [15,16]. Notter et al. [15] further reported that the immunofluorescence detection sensitivity of postsynaptic receptors (such as the $\mathrm{GABA}_{\mathrm{A}}$ receptor $\alpha 2$ ) in brain tissues largely depends on the strength of the fixation, which is mostly determined by fixation duration. We therefore adopted this method (a brief, weak immersion-fixation) to achieve our aim of detecting GluA1 receptors in the PSD area of the NAcc.

A previous study by Schneider et al. [16] showed that GluA1 was detected as an intracellular distribution even with a weak immersion-fixation protocol similar to ours. One major reason for this discrepancy could be the thickness of the tissue block prepared for immersion-fixation. Schneider et al. [16] used 1-2 mm thick tissue blocks, while our tissue blocks were 9-10 mm wide. Because the strength of fixation of a tissue immersed in aldehyde solution can be influenced by different sample thicknesses, a strong fixation might be achieved for relatively thin tissue samples even with a short period of fixation. In our case, the detection of GluAl sensitivity was obviously enhanced by a brief immersion-fixation. It is important to note, however, that this protocol cannot be generalized and, in order to achieve labeling efficiency, researchers should adjust the protocol appropriately, focusing on key factors such as duration of fixation depending on the nature of the tissue sample. We also emphasize here that our protocol is not aimed at replacing conventional perfusion-fixation, which is still a gold standard and suitable method for most immunohistochemistry applications. Instead, the immersionfixation method provides a feasible solution for the detection of postsynaptic proteins, which are hard to detect with conventional perfusion protocols.

The AMPA receptors are an important part of the process of adaptation to chronic cocaine exposure. Biochemical analyses 
have revealed that surface expression of the GluA1 subunit gradually increases during withdrawal periods (as observed on days 7 through 21 with non-contingent cocaine exposure) in the NAcc core and shell $[11,28]$. Consistent with these findings, we detected a significant increase in GluA1 levels in two sub-regions of the NAcc on withdrawal day 21 in cocaine- compared to salineexposed rats. Our data, collected using a new methodological approach, confirm previous findings that AMPA receptors are differentially up-regulated during long-term withdrawal periods (day 21) after chronic cocaine exposure. Furthermore, by co-labeling PSD95, we were able to focus our analyses to directly measure GluA1 levels in the PSD area, and our results were similar to those from previous studies that measured GluA1 levels at the cell surface $[6,11]$.

One limitation of this study is that we did not examine other subunits of AMPA receptors. Our primary aim to determine whether the immersion-fixation technique would allow successful detection of AMPA receptors in the PSD area of the NAcc. For this purpose, we selected GluA1 as a representative subunit for our preliminary experiments but we do not yet know how the increased levels of the GluA1 subunit contribute to AMPAmediated synaptic strength in the NAcc. One possibility may be via the GluA2 subunit, which is known to regulate the calcium permeability of the AMPA receptors, which is another important measure of functional AMPA plasticity. Thus, the increase in GluA1 may contribute to potentiating AMPA-mediated fast neurotransmission in the NAcc either via a heterogeneous combination with GluA2 (calcium impermeable) or homogeneous one with GluAl alone (calcium permeable) [19,33]. In a future study, we plan to examine the levels and localization of GluA2 using the same approach employed in the present investigation.

One of the advantages of immunohistochemistry is the ability to localize proteins in situ with high spatial resolution. By using various sensitive probes combined with high-resolution imaging techniques, we can visualize and quantify proteins originating from different cell types and sub-cellular areas. Such morphological methods, in addition to widely used biochemical and electrophysiological measures, will help to elucidate the molecular organization of synapses more precisely, and expand our understanding of neuronal plasticity and brain function. In the present study, we used a modified immunohistochemical protocol to demonstrate differential changes in the GluA1 subunit of AMPA receptors in the NAcc during the long-term withdrawal period after cocaine exposure. We believe that our results hold promise for expanding our understanding of synaptic molecular plasticity through morphological approaches using immunohistochemistry and imaging techniques.

\section{ACKNOWLEDGEMENTS}

This work was supported by the National Research Foundation of
Korea (NRF) grants (2018R1A4A102523013; 2019R1A2C1011262) funded by the Korean government (MSIT).

\section{CONFLICTS OF INTEREST}

The authors declare no conflicts of interest.

\section{REFERENCES}

1. Kalivas PW, Volkow ND. The neural basis of addiction: a pathology of motivation and choice. Am J Psychiatry. 2005;162:1403-1413.

2. Goto Y, Grace AA. Limbic and cortical information processing in the nucleus accumbens. Trends Neurosci. 2008;31:552-558.

3. Groenewegen HJ, Wright CI, Beijer AV, Voorn P. Convergence and segregation of ventral striatal inputs and outputs. Ann N Y Acad Sci. 1999;877:49-63.

4. Mameli M, Halbout B, Creton C, Engblom D, Parkitna JR, Spanagel $\mathrm{R}$, Lüscher C. Cocaine-evoked synaptic plasticity: persistence in the VTA triggers adaptations in the NAc. Nat Neurosci. 2009;12:10361041.

5. Pierce RC, Wolf ME. Psychostimulant-induced neuroadaptations in nucleus accumbens AMPA receptor transmission. Cold Spring Harb Perspect Med. 2013;3:a012021.

6. Conrad KL, Tseng KY, Uejima JL, Reimers JM, Heng LJ, Shaham Y, Marinelli M, Wolf ME. Formation of accumbens GluR2-lacking AMPA receptors mediates incubation of cocaine craving. Nature. 2008;454:118-121.

7. Wolf ME, Ferrario CR. AMPA receptor plasticity in the nucleus accumbens after repeated exposure to cocaine. Neurosci Biobehav Rev. 2010;35:185-211.

8. Wolf ME, Tseng KY. Calcium-permeable AMPA receptors in the VTA and nucleus accumbens after cocaine exposure: when, how, and why? Front Mol Neurosci. 2012;5:72.

9. Pierce RC, Bell K, Duffy P, Kalivas PW. Repeated cocaine augments excitatory amino acid transmission in the nucleus accumbens only in rats having developed behavioral sensitization. J Neurosci. 1996;16:1550-1560.

10. Bäckström P, Hyytiä P. Involvement of AMPA/kainate, NMDA, and mGlu5 receptors in the nucleus accumbens core in cue-induced reinstatement of cocaine seeking in rats. Psychopharmacology. 2007; 192:571-580.

11. Boudreau AC, Wolf ME. Behavioral sensitization to cocaine is associated with increased AMPA receptor surface expression in the nucleus accumbens. J Neurosci. 2005;25:9144-9151.

12. Jedynak J, Hearing M, Ingebretson A, Ebner SR, Kelly M, Fischer RA, Kourrich S, Thomas MJ. Cocaine and amphetamine induce overlapping but distinct patterns of AMPAR plasticity in nucleus accumbens medium spiny neurons. Neuropsychopharmacology. 2016;41:464-476.

13. Harms KJ, Tovar KR, Craig AM. Synapse-specific regulation of AMPA receptor subunit composition by activity. J Neurosci. 2005;25:6379-6388.

14. Melone M, Burette A, Weinberg RJ. Light microscopic identification and immunocytochemical characterization of glutamatergic syn- 
apses in brain sections. J Comp Neurol. 2005;492:495-509.

15. Notter T, Panzanelli P, Pfister S, Mircsof D, Fritschy JM. A protocol for concurrent high-quality immunohistochemical and biochemical analyses in adult mouse central nervous system. Eur J Neurosci. 2014;39:165-175.

16. Schneider Gasser EM, Straub CJ, Panzanelli P, Weinmann O, Sassoè-Pognetto M, Fritschy JM. Immunofluorescence in brain sections: simultaneous detection of presynaptic and postsynaptic proteins in identified neurons. Nat Protoc. 2006;1:1887-1897.

17. Fritschy JM, Weinmann O, Wenzel A, Benke D. Synapse-specific localization of NMDA and GABA(A) receptor subunits revealed by antigen-retrieval immunohistochemistry. J Comp Neurol. 1998; 390:194-210.

18. Watanabe M, Fukaya M, Sakimura K, Manabe T, Mishina M, Inoue Y. Selective scarcity of NMDA receptor channel subunits in the stratum lucidum (mossy fibre-recipient layer) of the mouse hippocampal CA3 subfield. Eur J Neurosci. 1998;10:478-487.

19. Henley JM, Wilkinson KA. Synaptic AMPA receptor composition in development, plasticity and disease. Nat Rev Neurosci. 2016; 17:337-350.

20. Reimers JM, Milovanovic M, Wolf ME. Quantitative analysis of AMPA receptor subunit composition in addiction-related brain regions. Brain Res. 2011;1367:223-233.

21. Malinow R, Mainen ZF, Hayashi Y. LTP mechanisms: from silence to four-lane traffic. Curr Opin Neurobiol. 2000;10:352-357.

22. Santos SD, Carvalho AL, Caldeira MV, Duarte CB. Regulation of AMPA receptors and synaptic plasticity. Neuroscience. 2009;158:105-125.

23. Anderson SM, Famous KR, Sadri-Vakili G, Kumaresan V, Schmidt HD, Bass CE, Terwilliger EF, Cha JH, Pierce RC. CaMKII: a biochemical bridge linking accumbens dopamine and glutamate systems in cocaine seeking. Nat Neurosci. 2008;11:344-353.
24. Paxinos G, Watson C. The rat brain in stereotaxic coordinates. 5th ed. London: Elsevier Academic Press; 2004.

25. Sheng M, Kim E. The postsynaptic organization of synapses. Cold Spring Harb Perspect Biol. 2011;3:a005678.

26. Yoon HS, Kim S, Park HK, Kim JH. Microinjection of CART peptide 55-102 into the nucleus accumbens blocks both the expression of behavioral sensitization and ERK phosphorylation by cocaine. Neuropharmacology. 2007;53:344-351.

27. Kim WY, Jang JK, Lee JW, Jang H, Kim JH. Decrease of GSK3 $\beta$ phosphorylation in the rat nucleus accumbens core enhances cocaine-induced hyper-locomotor activity. J Neurochem. 2013;125: 642-648.

28. Ferrario CR, Li X, Wang X, Reimers JM, Uejima JL, Wolf ME. The role of glutamate receptor redistribution in locomotor sensitization to cocaine. Neuropsychopharmacology. 2010;35:818-833.

29. Malinow R, Malenka RC. AMPA receptor trafficking and synaptic plasticity. Annu Rev Neurosci. 2002;25:103-126.

30. Triller A, Choquet D. Surface trafficking of receptors between synaptic and extrasynaptic membranes: and yet they do move! Trends Neurosci. 2005;28:133-139.

31. Sassoé-Pognetto M, Utvik JK, Camoletto P, Watanabe M, Stephenson FA, Bredt DS, Ottersen OP. Organization of postsynaptic density proteins and glutamate receptors in axodendritic and dendrodendritic synapses of the rat olfactory bulb. J Comp Neurol. 2003;463:237-248.

32. Wouterlood FG, Böckers T, Witter MP. Synaptic contacts between identified neurons visualized in the confocal laser scanning microscope. Neuroanatomical tracing combined with immunofluorescence detection of post-synaptic density proteins and target neuronmarkers. J Neurosci Methods. 2003;128:129-142.

33. Wolf ME. Synaptic mechanisms underlying persistent cocaine craving. Nat Rev Neurosci. 2016;17:351-365. 